\title{
Kompakter Aufbau eines lichtadressiebaren potentiometrischen Sensors mit verfahrbarem Diodenlaser
}

\author{
Carl Frederik Werner ${ }^{1,2}$, Ahmed Mansour ${ }^{1}$, Franz-Matthias Rateike ${ }^{3}$, Sebastian Schusser ${ }^{1,2}$, Torsten \\ Wagner ${ }^{4}$, Tatsuo Yoshinobu ${ }^{4}$, Michael Keusgen5, Michael J. Schöning ${ }^{1,2}$ \\ ${ }^{1}$ Institut für Nano- und Biotechnologien (INB), FH Aachen, Campus Jülich, Heinrich-Mußmann- \\ Straße 1, 52428 Jülich \\ 2 Peter Grünberg Institut (PGI-8), Forschungszentrum Jülich GmbH, Wilhelm-Johnen-Straße, \\ 52525 Jülich \\ ${ }^{3}$ Labor für Lasertechnik, FH Aachen, Campus Jülich, Heinrich-Mußmann-Straße 1, 52428 Jülich \\ ${ }^{4}$ Lehrstuhl für Elektrotechnik, Tohoku Universität, 6-6-05 Aza Aoba, Aramaki, Aoba-ku, Sendai, \\ Miyagi 980-8579, Japan \\ ${ }^{5}$ Institut für pharmazeutische Chemie, Philipps Universität Marburg, Marbacher Weg 6-10, 35032 \\ Marburg
}

\section{Zusammenfassung}

Mit einem lichtadressierbaren potentiometrischen Sensor (LAPS) kann die Konzentration einer oder mehrerer Analyte in einer Lösung nachgewiesen werden. Dazu wird der Sensorchip mit einem modulierten Lichtzeiger abgerastert und der entstehende Fotostrom aufgezeichnet. Es wurde ein kompakter und einfacher LAPS-Aufbau mithilfe eines Diodenlasers entwickelt, welcher über piezoelektrische Linearmotoren bewegt wird. Die Erfassung des Fotostroms geschieht zeitgleich mit dem Modulationssignal, sodass gleichzeitig Amplitudenänderungen und Phasenverschiebung ermittelt werden können. Die Ortsauflösung wurde in der Fotostromamplitude und -phase nachgewiesen und die Auflösungsgrenze liegt bei ca. $250 \mu \mathrm{m}$.

\section{Einleitung}

Ein lichtadressierbarer potentiometrischer Sensor (LAPS) ist ein Halbleiter-basierter Chemo- bzw. Biosensor. Mit einem LAPS kann, je nach Oberflächenbeschichtung, ortsaufgelöst die Konzentration einer oder mehrerer Analyte in einer Lösung nachgewiesen werden [1]. Wie in Abbildung 1 zu sehen, besteht der Sensorchip eines LAPS aus Silizium mit einer Isolatorschicht. Auf dieser ist die sensitive Schicht, die in direktem Kontakt mit der Analytlösung steht, abgeschieden. Über eine Referenzelektrode und einen Rückseitenkontakt wird eine Bias-Spannung über diese Struktur angelegt. Mithilfe einer modulierten Lichtquelle wird gezielt in das Silizium geleuchtet. Dadurch entsteht ein Fotostrom, der abhängig von dem Oberflächenpotenzial an der beleuchteten Stelle, und somit von der lokalen Konzentration an der Sensoroberfläche, ist. Es gibt mehrere Ansätze, mit denen die Lichtquelle realisiert werden kann. Über Laser mit verfahrbarer Optik [2,3] oder mit Leuchtdioden (LED), die in einem Array verschaltet sind [4]. Zur Erzielung einer hohen Auflösung ist eine Lichtquelle mit kleinem Strahldurchmesser, mit dem der LAPSChip abgerastert werden kann, erforderlich. Um Streuungen in der Analytlösung sowie eine Beeinflussung dieser durch die Lichtquelle zu vermeiden, ist eine Einkopplung des Lichts an der LAPS-Chiprückseite zu bevorzugen.

Ähnlich wie in [3] wurde ein kompakter und einfacher LAPS-Aufbau mithilfe eines Diodenlasers entwickelt. Die Bewegung des Lasers erfolgt über piezoelektrische Linearmotoren. Die Erfassung des Fotostroms geschieht zeitgleich mit dem Modulationssignal, sodass Amplitudenänderungen und Phasenverschiebung ermittelt werden können. Somit ist es auch möglich, Messungen im kürzlich vorgestellten Phase-Mode [5] durchzuführen, bei dem nicht die Amplitudenänderung, sondern die Phasenänderung des Fotostroms betrachtet wird. 


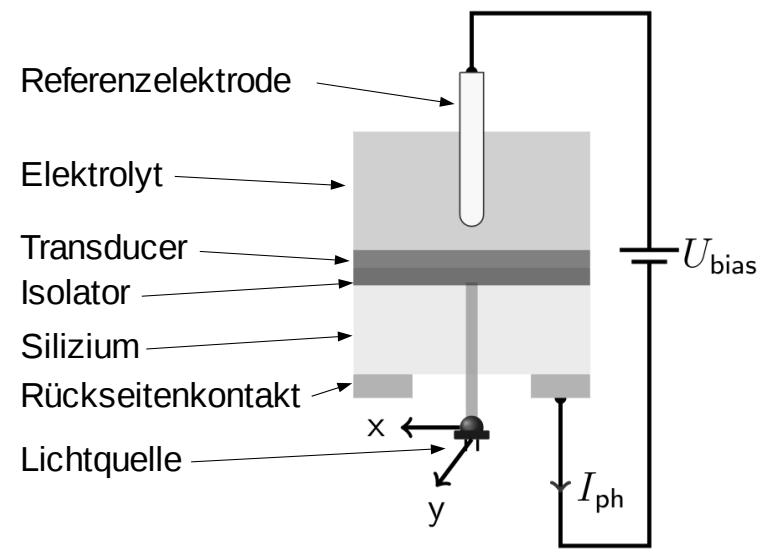

Bild 1 Schematische Darstellung eines lichtadressierbaren potentiometrischen Sensors (LAPS).

\section{Methoden und Aufbau}

Als Lichtquelle wurde ein Diodenlaser vom Typ QL78F6S-A der Firma QSI verwendet. Dieser Diodenlaser hat eine maximale Leistung von $10 \mathrm{~mW}$ und eine Wellenlänge von $780 \mathrm{~nm}$. Der Laser ist mit einer Linse auf einen Strahldurchmesser von ca. $70 \mu \mathrm{m}$ fokussiert. Betrieben wird dieser von einem Laserdiodentreiber ICWJ der Firma IC Haus. Damit ist es möglich, über einen Spannungseingang, die Laserleistung bis zu Frequenzen von $300 \mathrm{kHz}$ zu modulieren. Wie in Abbildung 2 zu sehen, ist der Diodenlaser auf zwei piezoelektrische Linearmotoren, die in einer XY-Anordnung verbaut sind, aufgesetzt. Die Nanomotoren der Firma Klocke haben einen Verfahrweg von \pm 9 mm bei einer Wiederholgenauigkeit von $40 \mathrm{~nm}$.

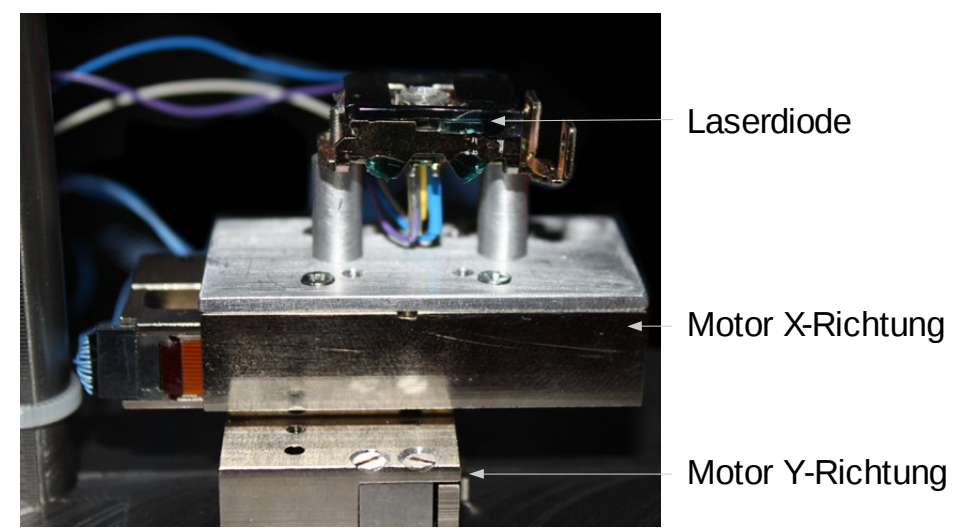

Bild 2 Die Nanomotoren in einer XY-Anordnung mit dem Diodenlaser.

Der Fotostrom wird von einem selbst gebauten Transimpedanzwandler verstärkt. Dieser verwendet den Operationsverstärker OPA656 der Firma Texas Instrument, hat einen Verstärkungsfaktor von $10^{6}$ V/A und wurde als Antialiasingfilter auf $120 \mathrm{kHz}$ beschränkt. Die Steuerung des Aufbaus erfolgt über eine eigens entwickelte LabVIEW-Software. Eine Datenerfassungskarte PCI MIO 16XE 10 von National Instruments dient zum Abtasten des verstärkten Fotostroms, Vorgeben der Bias-Spannung und Bereitstellen des Modulationssignals für den Diodenlasertreiber. Die Fotostromamplitude und -phase wird über eine schnelle Fouriertransformation (FFT) aus dem abgetasteten Signal berechnet. Somit lassen sich mit dem Aufbau Strom/Spannung (I/V) Kurven an vorgegebenen Positionen aufnehmen oder „Chemical Images“ erzeugen. Dabei wird bei einer festen Spannung der LAPS-Chip abgerastert und der resultierende Fotostrom bezüglich der Position in einem Falschfarbenbild dargestellt. 


\section{Ergebnisse}

Zur Charakterisierung des neu entwickelten Aufbaus wurden qualitative und quantitative Messungen zur Bestimmung der Ortsauflösung durchgeführt sowie eine Messung zur pH-Empfindlichkeit. Zur qualitativen Bestimmung wurde eine Polymerstruktur in Form eines „Smiley“ auf die Oberfläche aufgebracht. Die Aufnahme des „Chemical Image“ der LAPS-Struktur wurde bei einer Bias-Spannung von 0,2 V und pH 7 Pufferlösung durchgeführt. Die eingestellte Schrittweite der Nanomotoren betrug während der 28-minütigen Messung jeweils 0,2 mm. Das Ergebnis der Messung bezüglich der Fotostromamplitude (links) und -phase (rechts) ist in Abbildung 3 gezeigt. Bei beiden Messungen ist der „Smiley“ klar zu erkennen. Die Polymerschicht verhält sich wie eine zusätzliche Impedanz, weshalb die Fotostromamplitude an dieser Stelle sinkt. Ein Vergleich der Größen ergibt eine gute Übereinstimmung, so hat die Nase einen Durchmesser von 3,7 mm und aus dem „Chemical Image“ ergibt sich aus der Fotostromamplitude bzw. -phase ein Wert von $4 \mathrm{~mm}$.
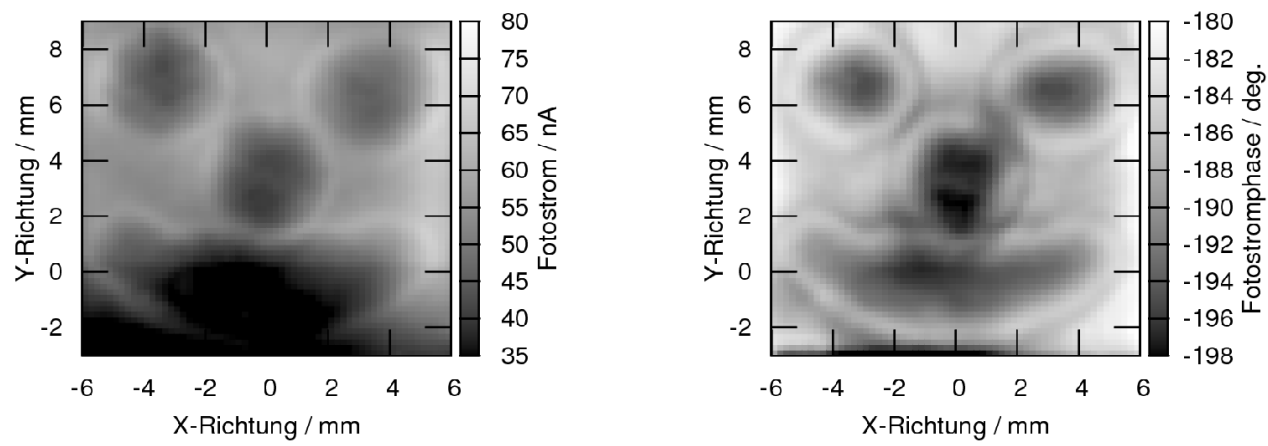

Bild 3 „Chemical Image“ eines LAPS-Chips mit aufgebrachter Polymerschicht in Form eines „Smiley“. Es ist die Fotostromamplitude (links) und -phase (rechts) abhängig von der Position gezeigt.

Zur quantitativen Bestimmung wurde eine LAPS-Struktur mit einer Siliziumdicke von $450 \mu \mathrm{m}$ und einer zusätzlichen Goldschicht benutzt. Diese Goldschicht wurde fotolithografisch so strukturiert, dass Linienstrukturen der Breite $100 \mu \mathrm{m}, 250 \mu \mathrm{m}, 500 \mu \mathrm{m}, 1 \mathrm{~mm}$ und $2 \mathrm{~mm}$ entstanden, ähnlich wie in [6] beschrieben. Zur Messung wurde die Goldstruktur anstelle der Referenzelektrode und des Elektrolyten kontaktiert. Die Messung dauerte insgesamt, bei einer Schrittweite von 0,1 mm, 1 Stunde und 40 Minuten. Abbildung 4 zeigt die Fotostromamplitude. Die Linienstrukturen mit einer Breite von $500 \mu \mathrm{m}, 1 \mathrm{~mm}$ und $2 \mathrm{~mm}$ sind gut zu erkennen. Die 250- $\mu \mathrm{m}$-Strukturen sind etwas unscharf und die 100- $\mu \mathrm{m}$-Linien können nicht mehr erkannt werden.

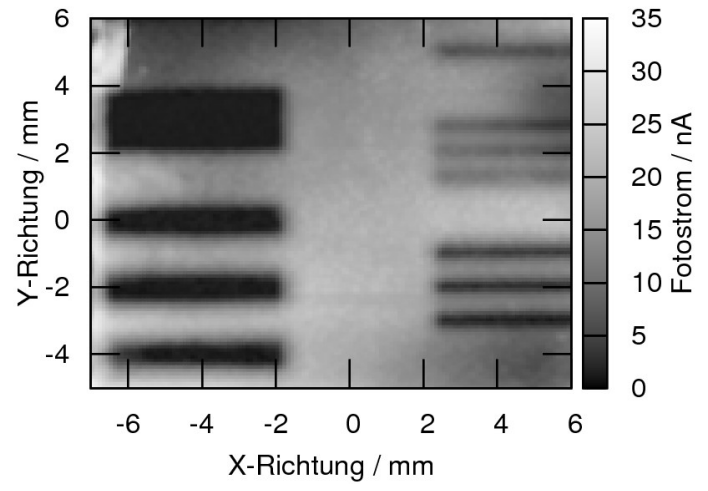

Bild 4 Fotostromamplitude bei Abrasterung einer LAPS-Struktur mit einer Goldschicht mit unterschiedlich breiten Linienstrukturen zur Bestimmung der Ortsauflösung. Die Linienstrukturen sind wie folgt angeordnet: 2 mm oben links, $1 \mathrm{~mm}$ unten links, $500 \mu \mathrm{m}$ unten rechts, $250 \mu \mathrm{m}$ Mitte rechts und $100 \mu \mathrm{m}$ oben rechts.

Abbildung 5 zeigt I/V-Kurven einer pH-empfindlichen LAPS-Struktur mit einer $\mathrm{Ta}_{2} \mathrm{O}_{5}$-Oberfläche, die an einer Stelle mit unterschiedlichen $\mathrm{pH}$-Pufferlösungen gemessen wurden. Es ist eine pH-Abhängigkeit aufgrund der Verschiebung der I/V-Kurven auf der Spannungsachse zu erkennen. Die pH-Sensitivität liegt bei einem Arbeitspunkt von $0,4 \mu \mathrm{A}$ mit $(58,0 \pm 1,9) \mathrm{mV} / \mathrm{pH}$ nahe dem theoretischen Wert von $59 \mathrm{mV} / \mathrm{pH}$. 

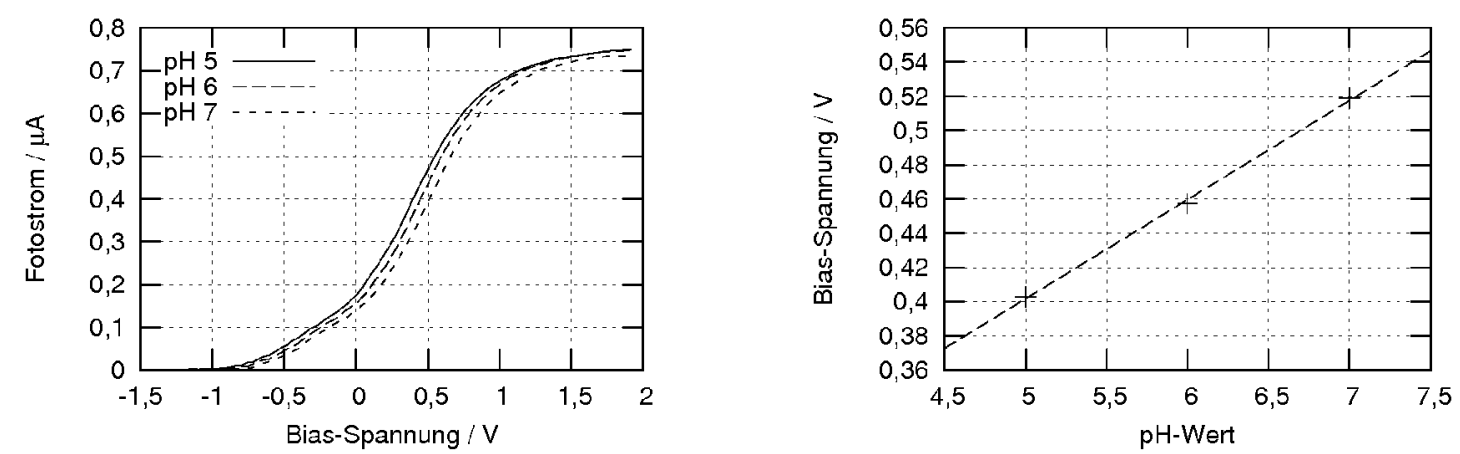

Bild 5 Strom/Spannung (I/V) Kurven bei unterschiedlichen pH-Pufferlösungen zwischen pH 5 und pH 7 (links) und die zugehörige Kalibrationsgerade (rechts).

\section{Diskussion und Ausblick}

Es wurde ein LAPS-Aufbau mithilfe einer Laserdiode und piezoelektrischen Linearmotoren mit einer Wiederholgenauigkeit von $40 \mathrm{~nm}$ entwickelt. Die Laserdiode ist mit der Linse direkt auf den Motoren montiert, was eine kompakte Bauform ermöglicht. Das Modulationssignal des Diodenlasers kann frei gewählt werden, um Frequenzen bis zu $120 \mathrm{kHz}$ enthalten. Bei einer Messung zur qualitativen Bestimmung der Ortsauflösung konnte eine, auf der LAPS-Struktur aufgebrachte Polymerschicht sowohl in der Fotostromamplitude als auch in der Fotostromphase nachgewiesen werden. Somit ist es auch möglich, Messungen im kürzlich vorgestellten Phase-Mode [5] durchzuführen. Bei einer Siliziumdicke von $450 \mu \mathrm{m}$ und einer Wellenlänge von $78 \mathrm{~nm}$ liegt die Auflösungsgrenze des Aufbaus bei ca. $250 \mu \mathrm{m}$, was sich mit Literaturangaben [6,7] deckt.

Mit diesem kompakten Aufbau kann die Konzentrationsverteilung in Lösungen untersucht werden oder die Konzentration mehrerer Analyte gleichzeitig nachgewiesen werden [8]. Es ist auch möglich, die metabolische Aktivität von Zellkulturen ortsaufgelöst zu untersuchen, indem die lokale pH-Wertänderung, die durch deren Metabolismus hervorgerufen wird, ermittelt wird [2].

\section{Danksagung}

Die Autoren danken dem BMELV und der Fachagentur Nachwachsende Rohstoffe e.V. für die finanzielle Förderung des Forschungsvorhabens („Bio-LAPS“).

\section{Literatur}

[1] Owicki, J. C.; Bousse, L. J.; Hafeman, D. G.; Kirk, G. L.; Olson, J. D.; Wada, H. G.; Parce, J. W., Annual Review of Biophysics and Biomolecular Structure, 23, 87-113 (1994)

[2] Yoshinobu, T.; Ecken, H.; Ismail, A. B. M.; Iwasaki, H.; Lüth, H.; Schöning, M. J., Electrochimica Acta, 47(1-2), 259-263 (2001)

[3] Moritz, W.; Gerhardt, I.; Roden, D.; Xu, M.; Krause, S., Fresenius' Journal of Analytical Chemistry, 367(4), 329-333 (2000)

[4] Wagner, T.; Molina, R.; Yoshinobu, T.; Kloock, J. P.; Biselli, M.; Canzoneri, M.; Schnitzler, T.; Schöning, M. J., Electrochimica Acta, 53(2), 305-311 (2007)

[5] Miyamoto, K.; Wagner, T.; Yoshinobu, T.; Kanoh, S.; Schöning, M. J., Sensors and Actuators, B: Chemical, 154(1), 28-32 (2011)

[6] Nakao, M.; Inoue, S.; Yoshinobu, T.; Iwasaki, H., Sensors and Actuators B: Chemical, 34, 234-239 (1996)

[7] George, M.; Parak, W. J.; Gerhardt, I.; Moritz, W.; Kaesen, F.; Geiger, H.; Eisele, I.; Gaub, H. E., Sensors and Actuators, A: Physical, 86, 187-196 (2000)

[8] Yoshinobu, T.; Iwasaki, H.; Ui, Y.; Furuichi, K.; Ermolenko, Y.; Mourzina, Y.; Wagner, T.; Näther, N.; Schöning, M. J., Methods, 37, 94-102 (2005) 